\title{
Supporting Information: Machine Learning Driven Analysis of Large Scale Simulations Reveals Conformational Characteristics of Ubiquitin Chains
}

\author{
Andrej Berg ${ }^{1, *}$, Leon Franke ${ }^{1}$, Martin Scheffner ${ }^{2}$, and Christine Peter ${ }^{1, *}$ \\ ${ }^{1}$ Department of Chemistry, University of Konstanz, Universitätsstraße 10, Konstanz 78457, \\ Germany \\ ${ }^{2}$ Department of Biology, University of Konstanz, Universitätsstraße 10, Konstanz 78457, \\ Germany \\ ${ }^{*}$ Corresponding author: andrej.berg@uni-konstanz.de, christine.peter@uni-konstanz.de
}

March 16, 2020

Supporting information with additional figures showing details about EncoderMap training, all two-dimensional representations obtained for diUb and triUb and details about HDBSCAN clustering. Numbering of figures according to main document to indicate thematic association. 


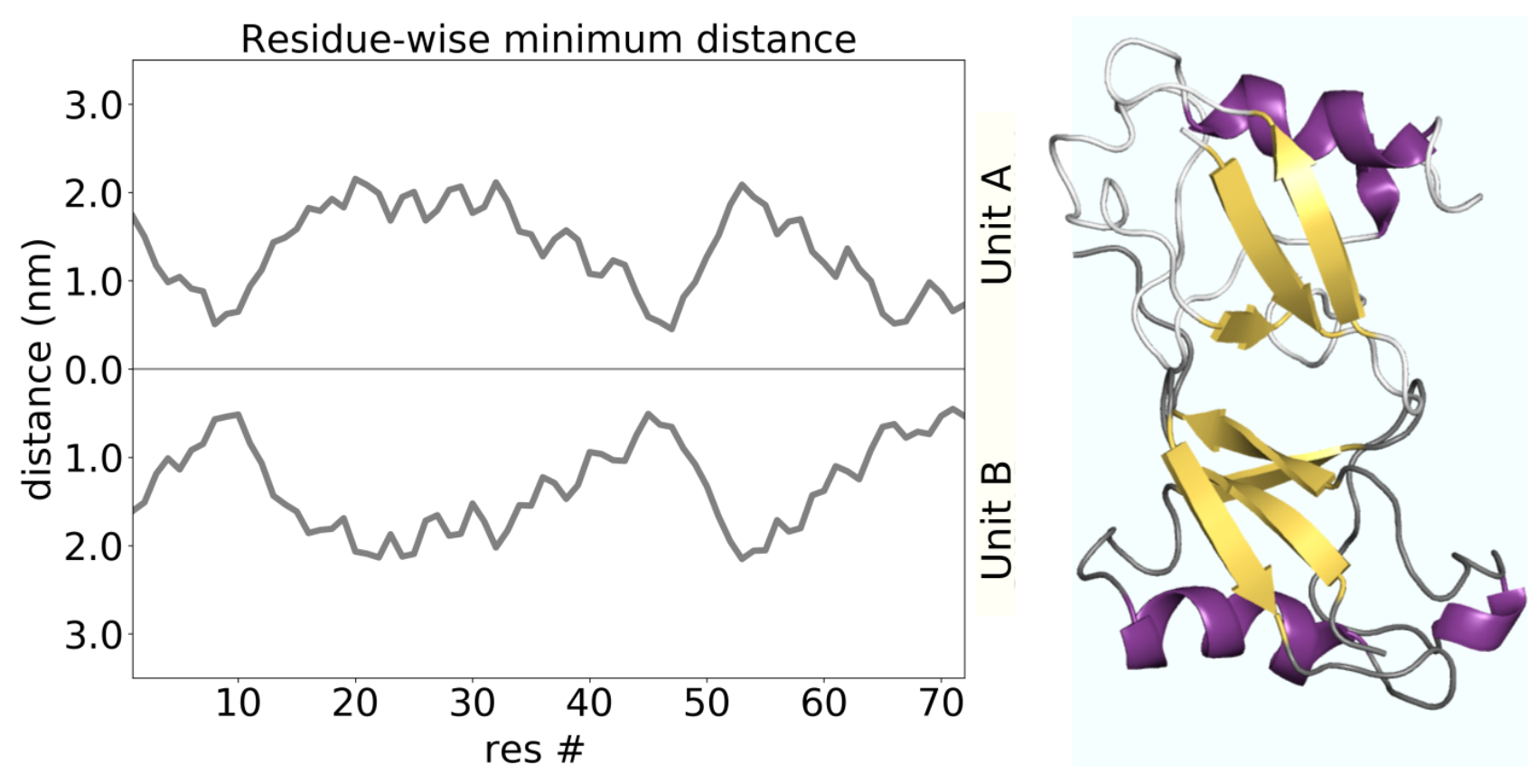

Figure S3.1: Collective variables. Illustration of residue-wise minimum distances (RMD) between two unlinked Ub proteins. Upper part of diagram shows the minimum distance for each residue in unit A to unit B. Lower part of diagram shows corresponding distances for residues in unit B with an inverted y-axis to give a butterfly plot. Corresponding $2 \mathrm{xUb}$ configuration on the right has a very point symmetric interaction interface.
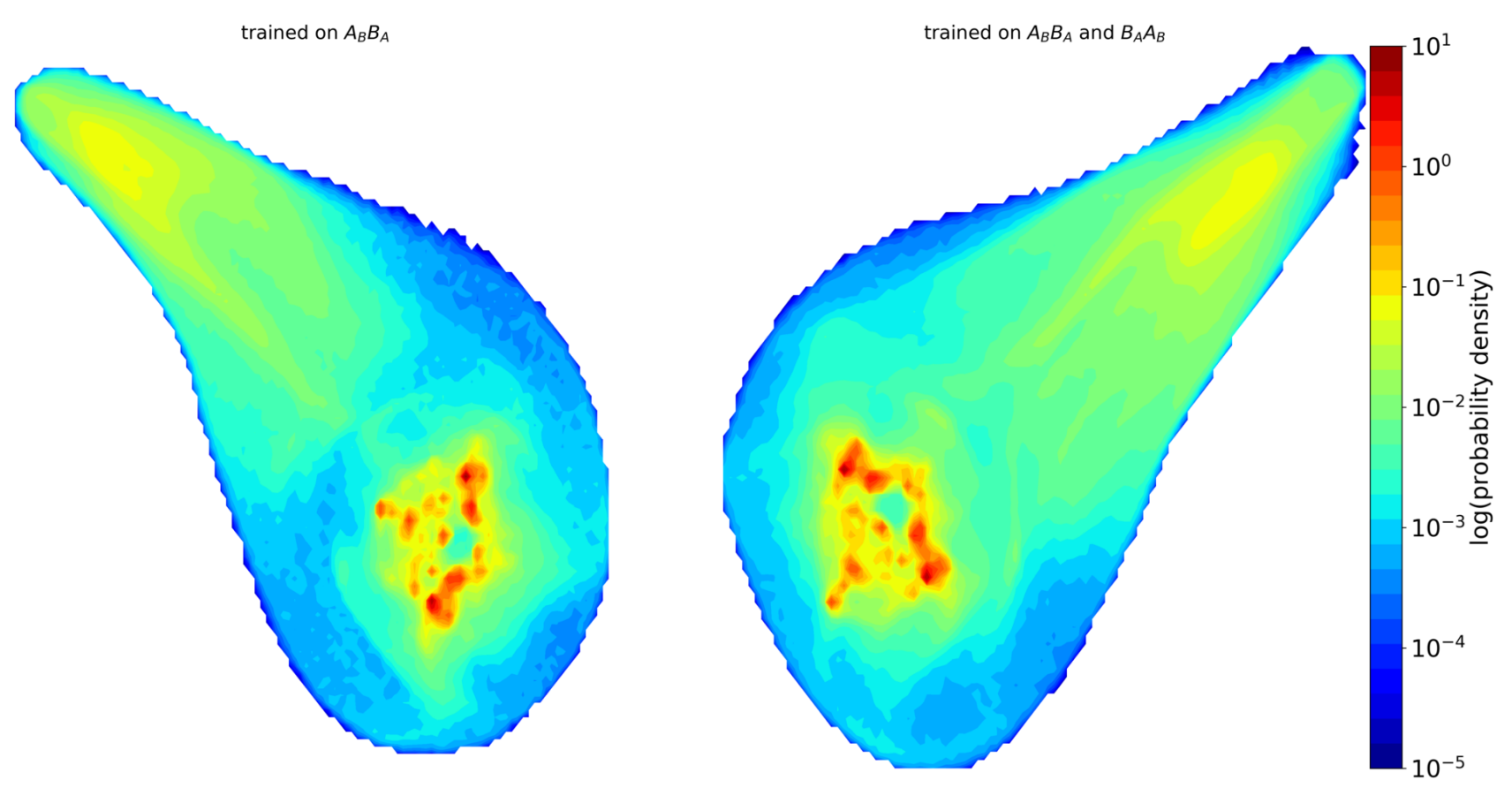

Figure S3.2: Impact of training on doubled data set. Left histogram shows EncoderMap projection of 2xUb configurations which was trained on a normal RMD data set $\left(A_{B} B_{A}\right)$. Right histogram shows EncoderMap projection which was used in this study and was trained on doubled/symmetrized RMD data set $\left(A_{B} B_{A}\right.$ and $\left.B_{A} A_{B}\right)$. Coloring according to the logarithm of the probability density. 

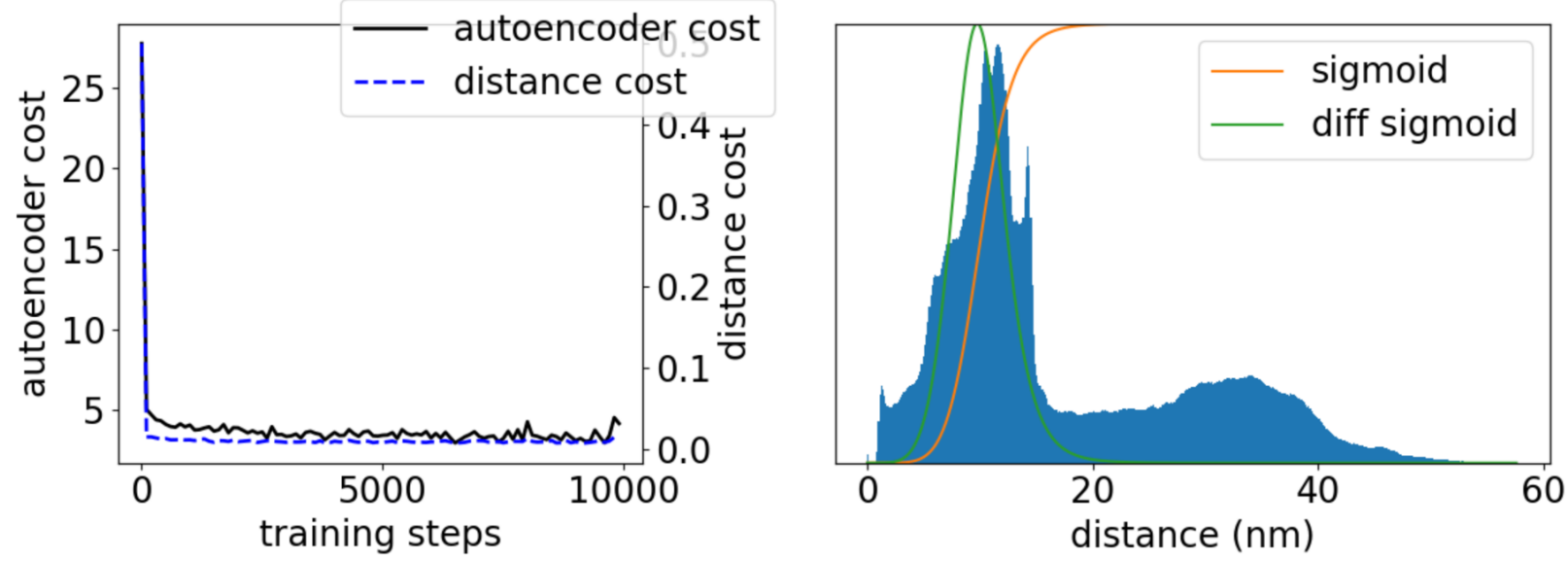

Figure S3.3: Illustration of parameters for sigmoid function used to train an EncoderMap neural network on RMD data from 2xUb CG simulations. Distance distribution (blue) of high-dimensional data (RMD, 144D) and resulting sigmoid function (orange) and its first derivative (green). Green graph indicates window where distances should be preserved during dimensionality reduction. 

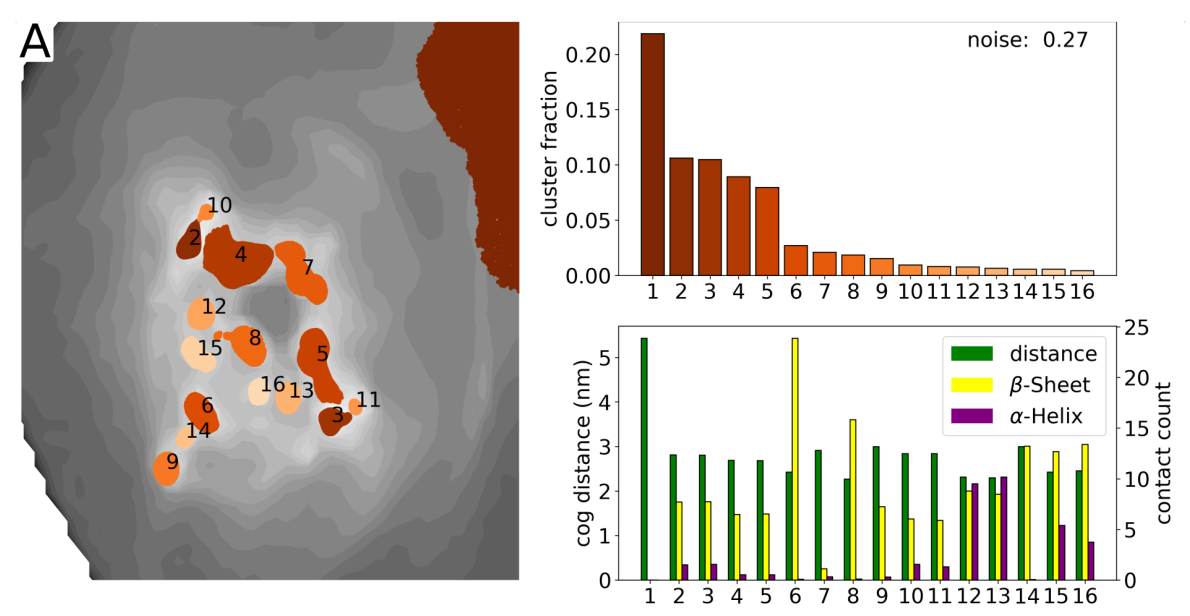

B
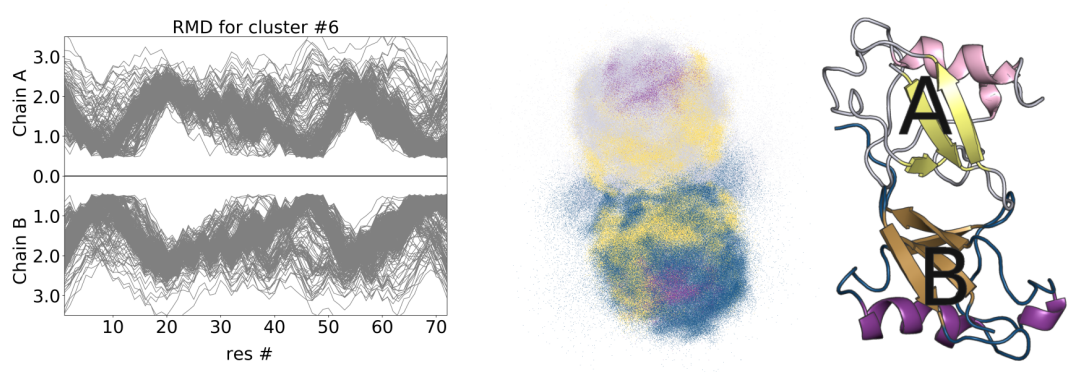

C
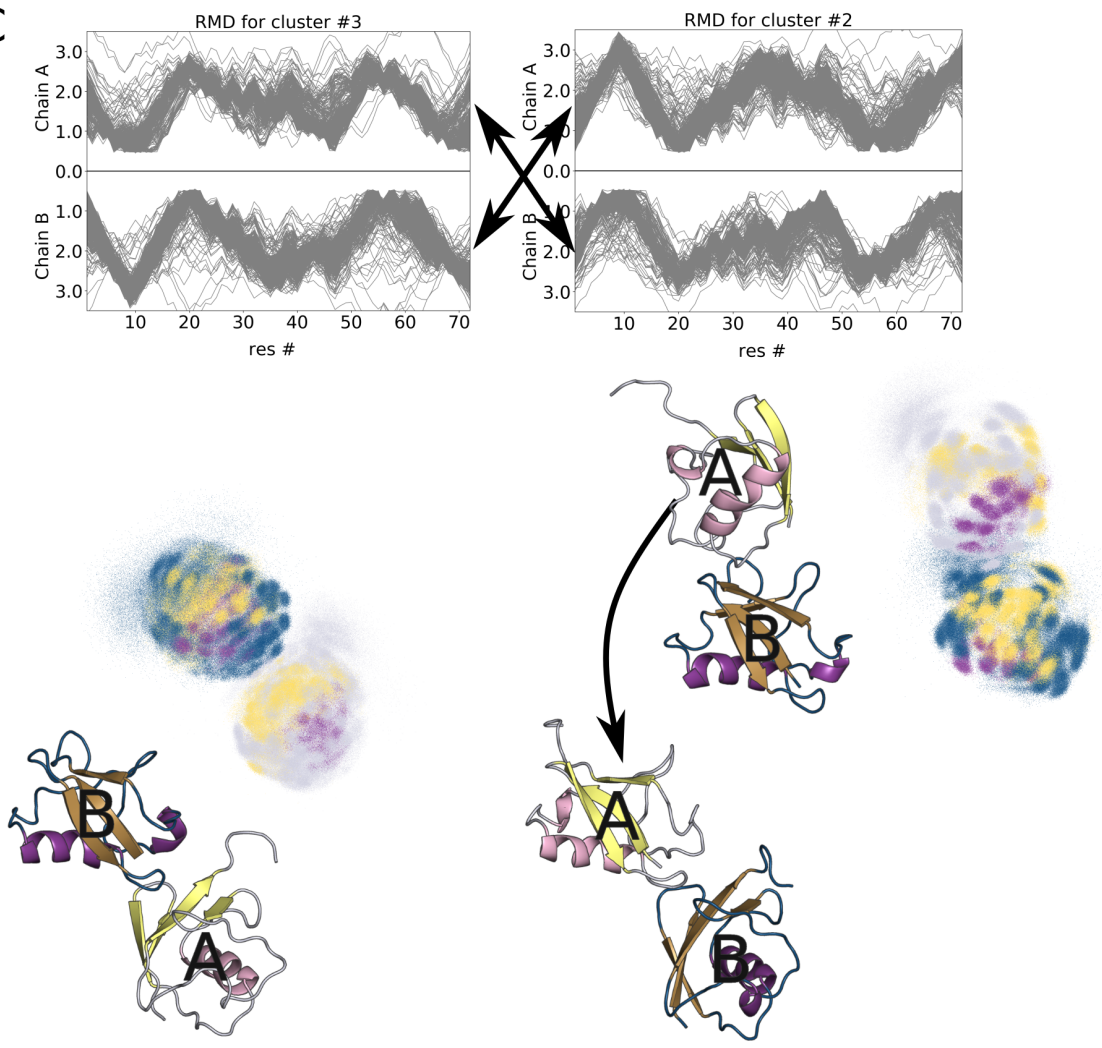

Figure S4.1: Illustration of cluster-mapping performed for 2xUb. A: Low-dimensional representation of clustered $2 \mathrm{xUb}$ configurations (orange scatter) on 2xUb landscape (gray histogram) with cluster ids. Right graphs show fractions and mean configurational properties for each cluster. B: RMD values as butterfly plot for $2 \mathrm{xUb}$ configurations which were assigned to cluster id 6 (left graph) and corresponding CG structure bundle with back-mapped (atomistic) representative configuration (cartoon). C: RMD values as butterfly plots for $2 \mathrm{xUb}$ configurations from two clusters (id 2 and id 3) which are symmetry equivalent. Similarity of parts of RMD vector is indicated by arrows. Below: illustration how representative configurations from these two clusters become almost identical if Ub unit order is exchanged. 


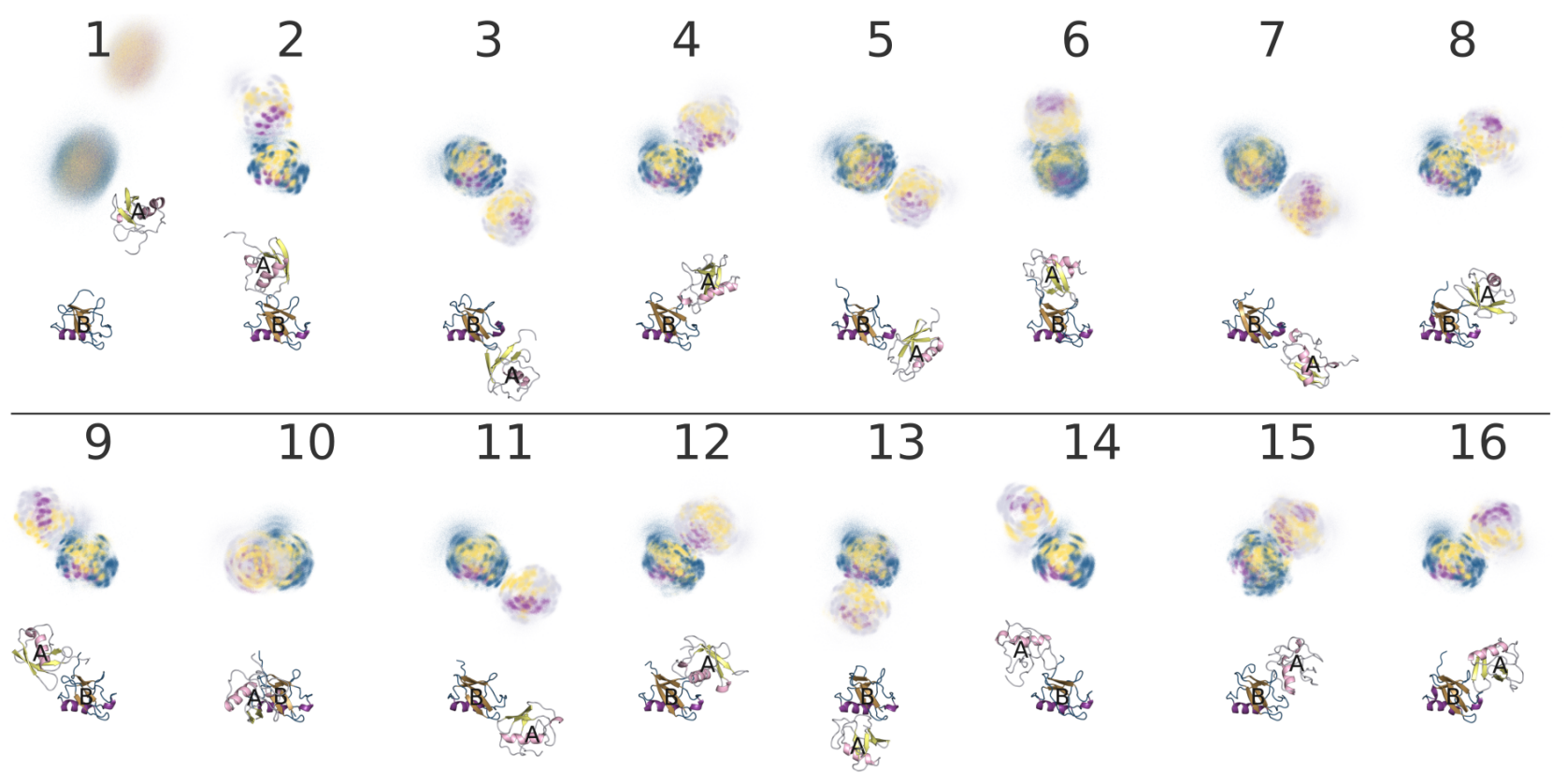

Figure S4.2: 2xUb configurations found by clustering. Structure bundles of CG structures (spheres) obtained from clustering with HDBSCAN. Structures were aligned to back bone beads of the representative configuration inside a cluster. Colored according to secondary structure assignment obtained from PDB-ID: 1UBQ. Cartoon representation shows representative configuration obtained by back-mapping to the atomistic level. Cluster ids according to Figure S4.1 as number above each representation.
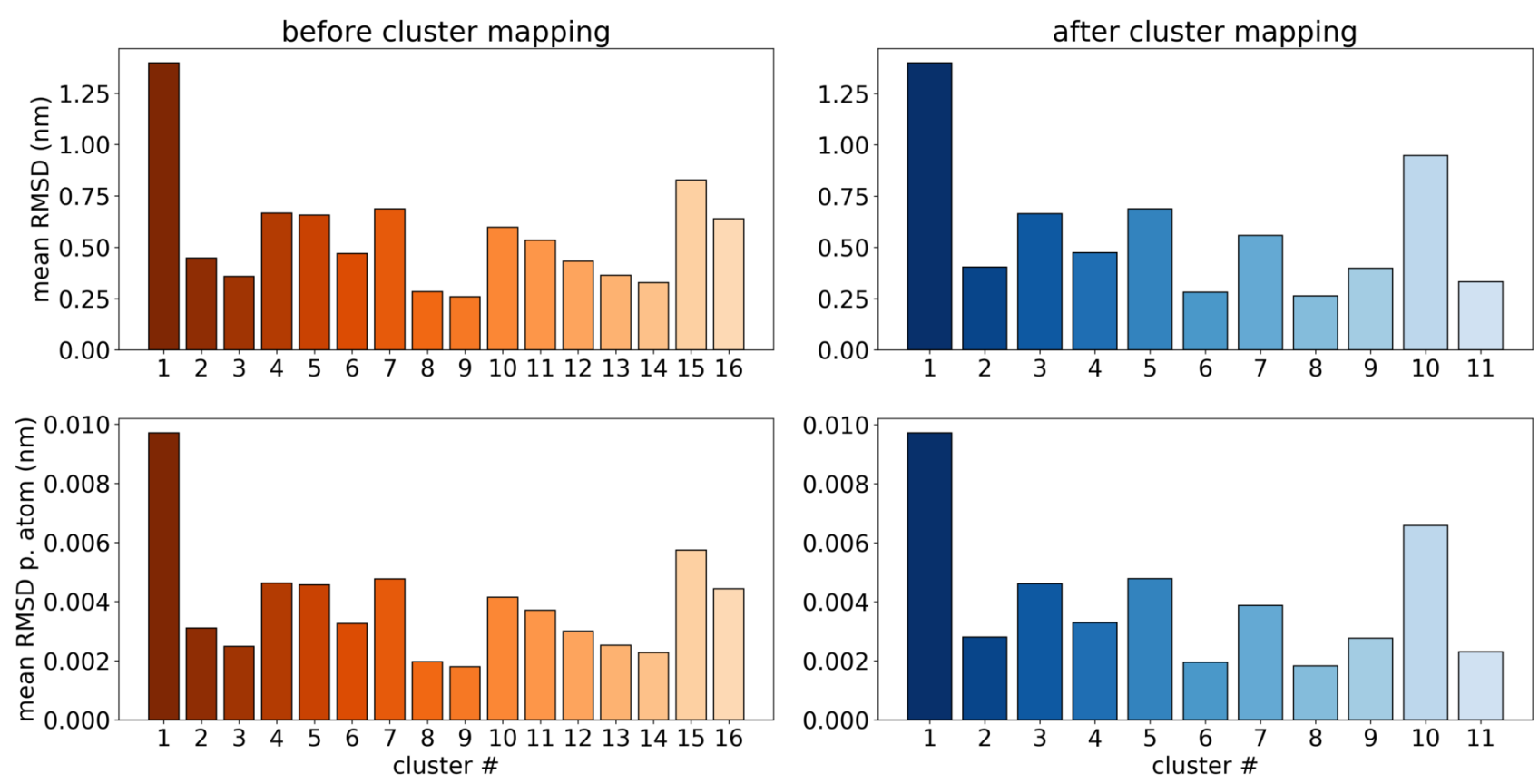

Figure S4.3: Mean RMSD values obtained from pair-wise RMSD matrix between conformations found by clustering. RMSD was calculated for those beads of the CG model, which were used to obtain RMD values (back-bone beads of residues 1 to 72 in each Ub protein). Left graphs (orange) show mean RMSD values calculated between $\mathrm{CG} 2 \mathrm{xUb}$ configurations which were clustered together initially by HDBSCAN (according to Figure S4.1). Right graphs (blue) show mean RMSD values calculated after cluster mapping of symmetry equivalent clusters (according to Figure 4). 

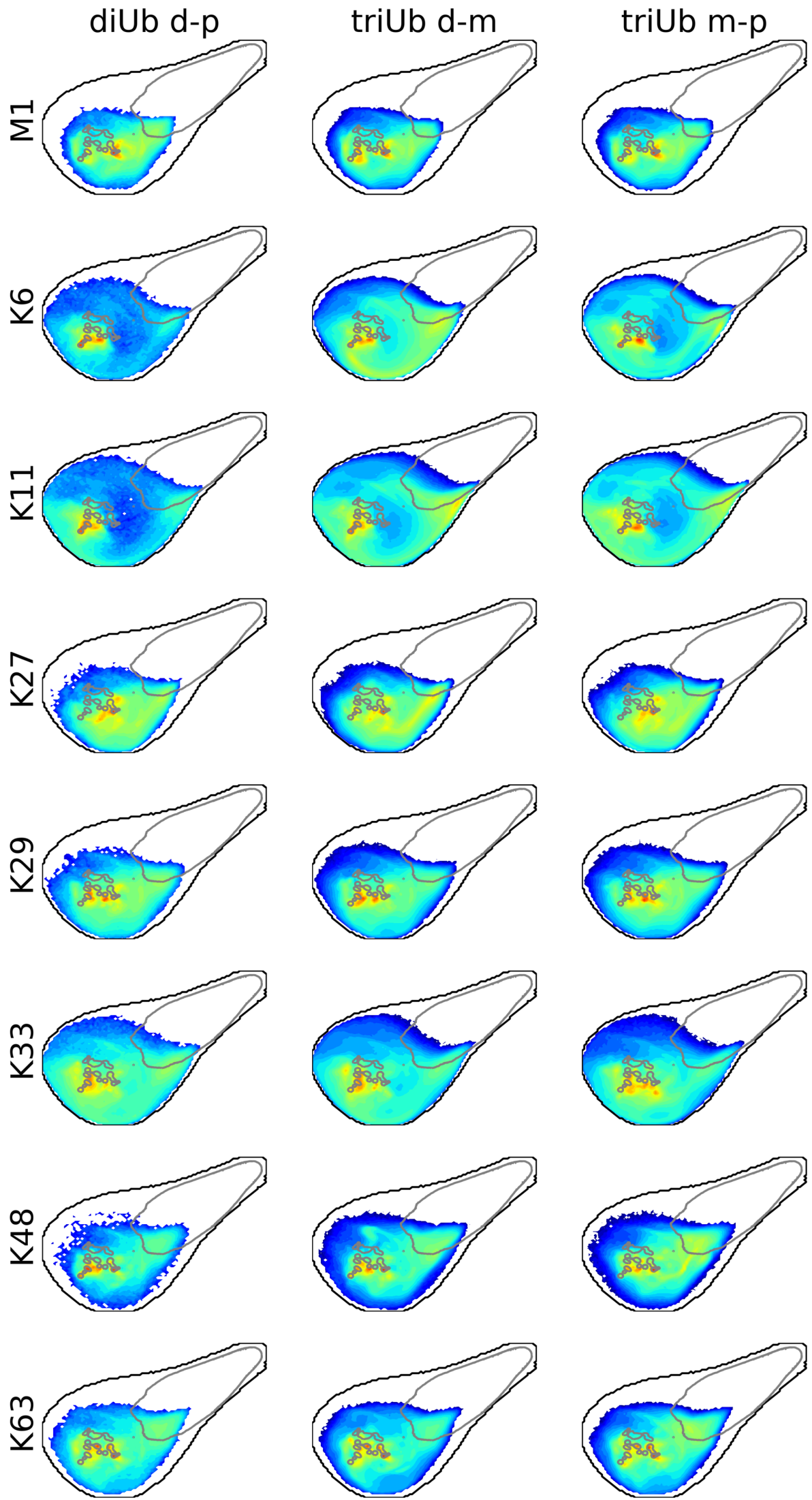

Figure S5.1: EncoderMap projections of diUb and triUb. Two-dimensional representations/landscapes separated by linkage type (colored histograms) with outer rim of $2 \mathrm{xUb}$ landscape (black) and outlines of clusters found for $2 \mathrm{xUb}$ (gray). Coloring according to the logarithm of the probability density. Color-bar according to Figure 5. Left column: conformational landscapes of diUb; middle and right column: interaction landscapes of triUb between distal and middle Ub moiety and middle and proximal moiety, respectively. 

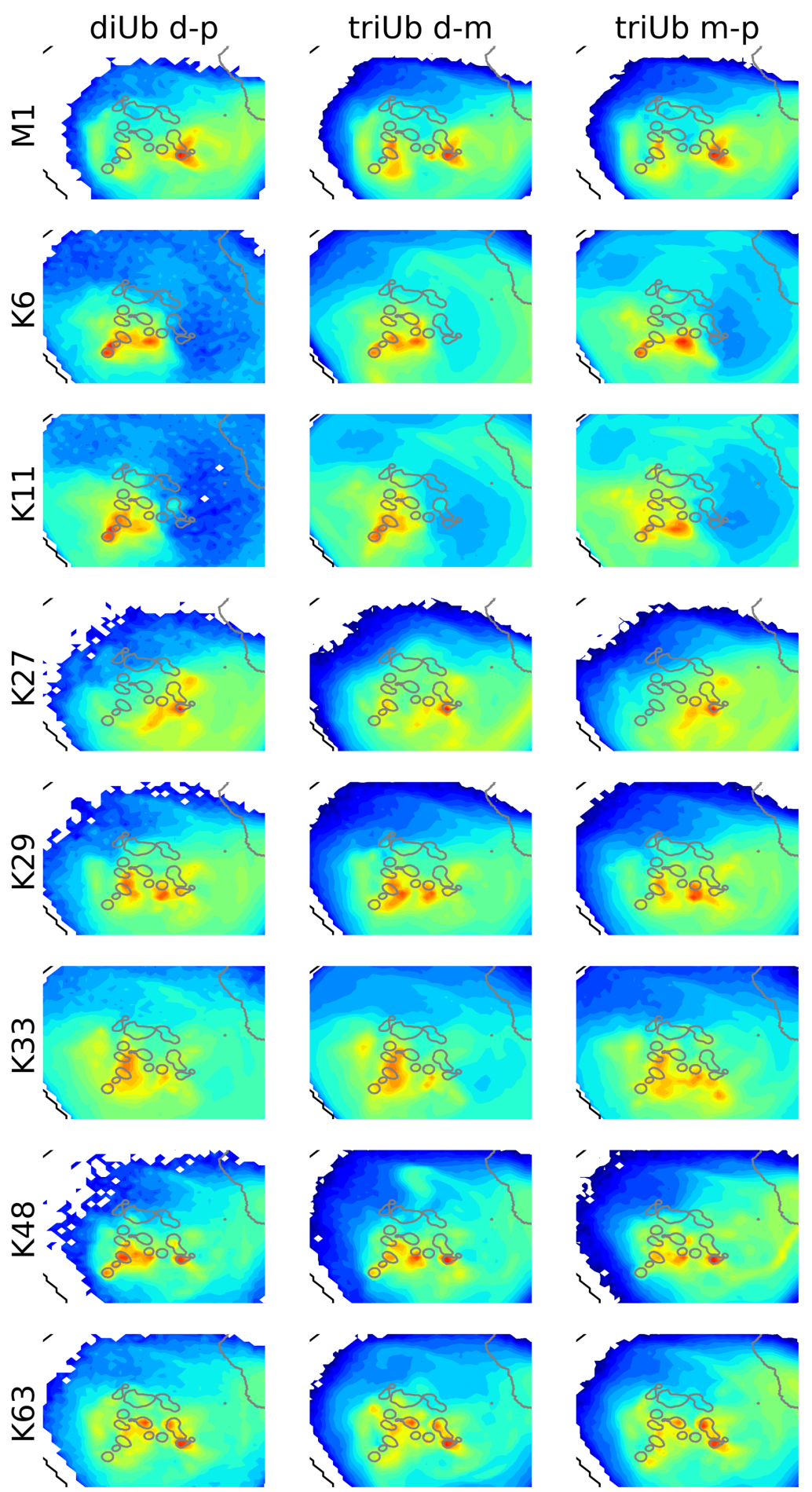

Figure S5.2: EncoderMap projections of diUb and triUb. Zoom into Figure S5.1. Two-dimensional representations/landscapes separated by linkage type (colored histograms) with outer rim of $2 x$ Ub landscape (black) and outlines of clusters found for $2 \mathrm{xUb}$ (gray). Coloring according to the logarithm of the probability density. Color-bar according to Figure 5. Left column: conformational landscapes of diUb; middle and right column: interaction landscapes of triUb between distal and middle Ub moiety and middle and proximal moiety, respectively. 


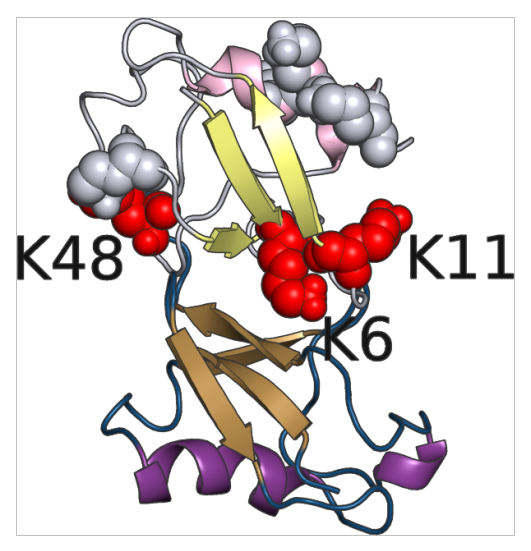

Figure S5.3: Representative conformation for an interface formed by K6, K11 and K48-linked diUb. Lysine residues are shown as spheres and K6, K11 and K48 are colored red, which illustrates their positions inside the interface. In consequence this interface is unfavorable in triUb where these lysine side-chains are linked to the next Ub moiety.
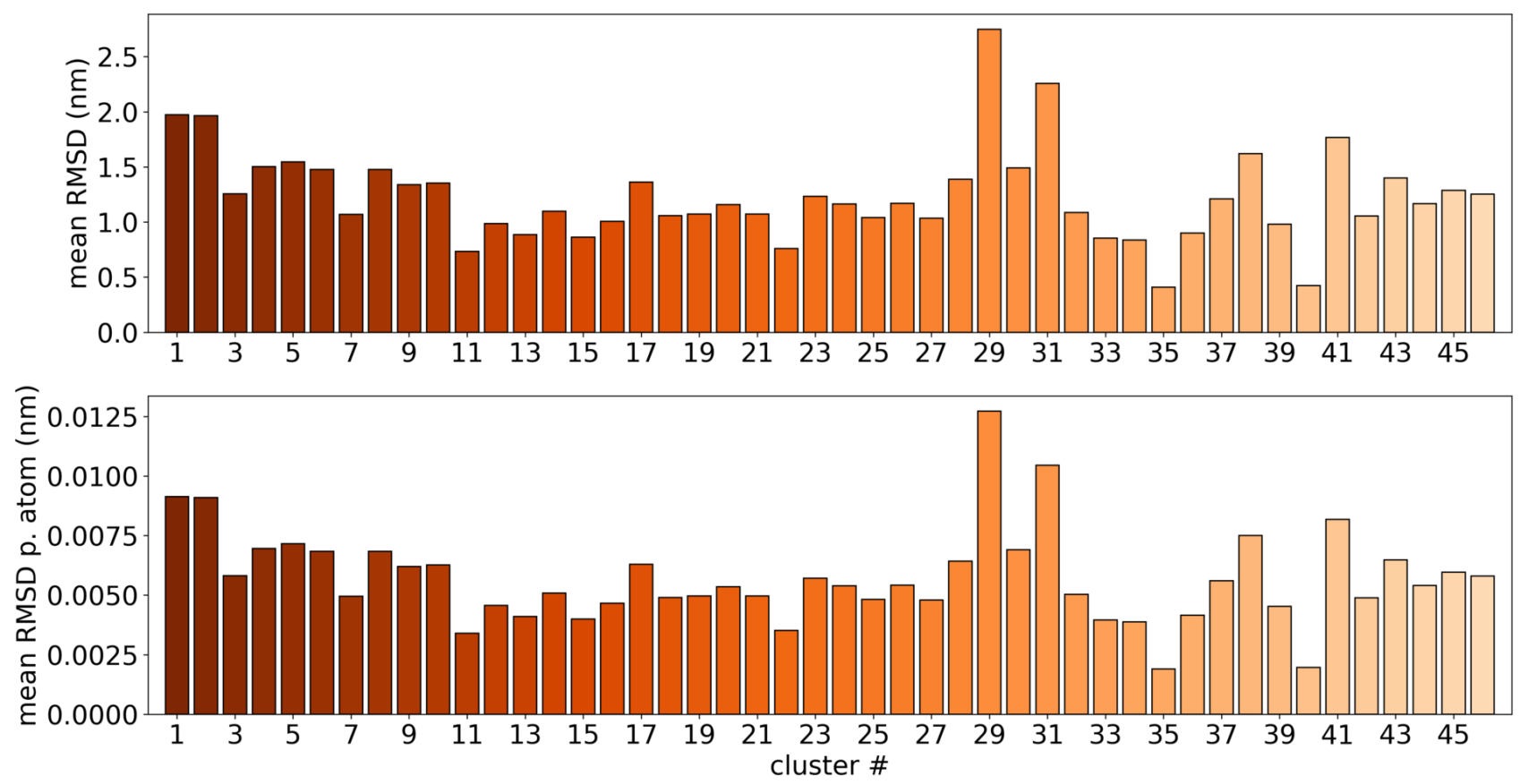

Figure S8.1: Mean RMSD values obtained from RMSD matrix between conformations found by HDBSCAN clustering of $3 \mathrm{xUb}$ configurations. RMSD was calculated for those beads of the CG model, which were used to obtain RMD values (back bone beads of residues 1 to 72 in each Ub protein, 216 beads). 

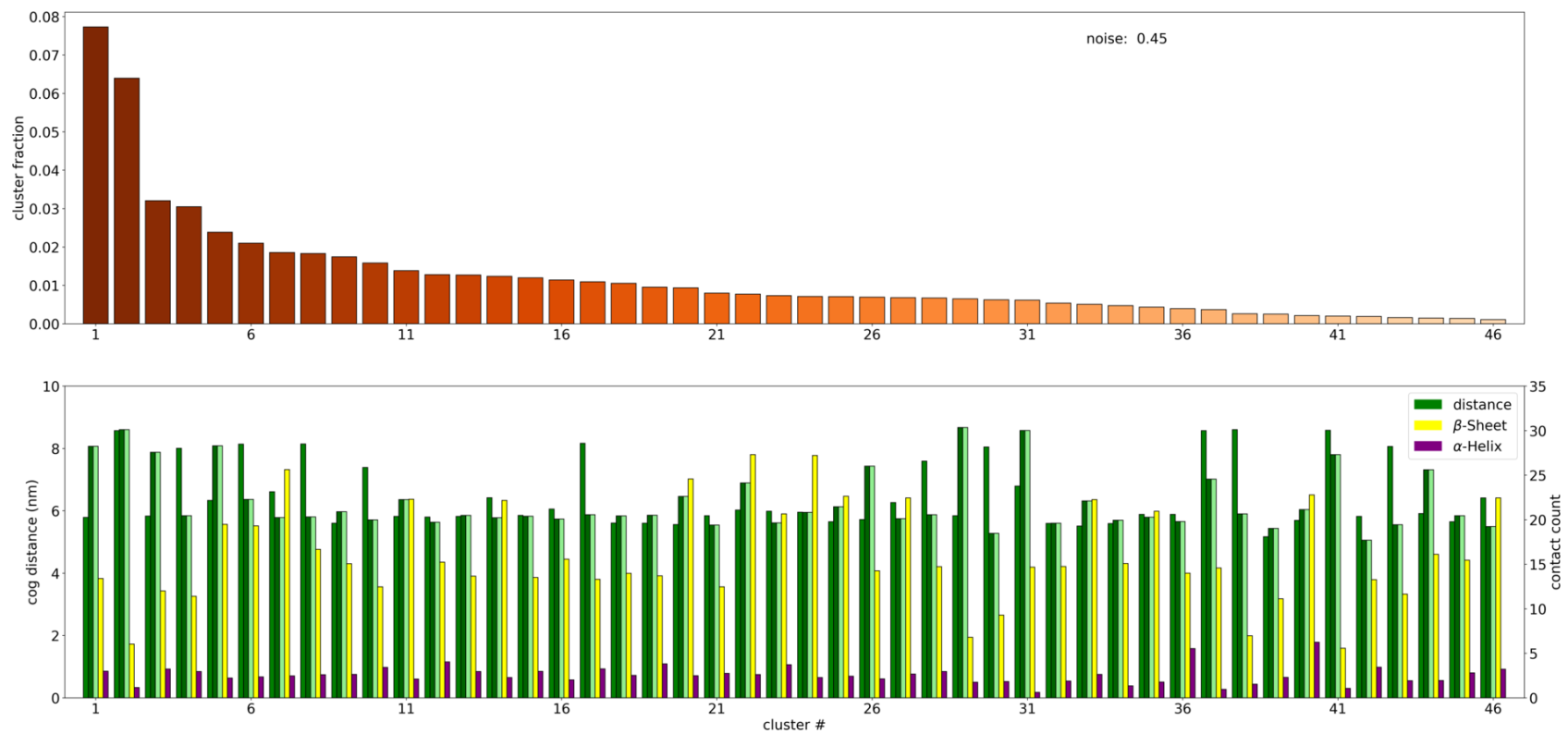

Figure S8.2: $3 \mathrm{xUb}$ cluster properties. Cluster fractions (orange) and mean configurational properties for clusters found by HDBSCAN for 3xUb. Mean center of geometry distance between Ub proteins (green). Mean contact count between Ub proteins evaluated for residues involved in a $\beta$-sheet (yellow) or $\alpha$-helix (purple) motif.
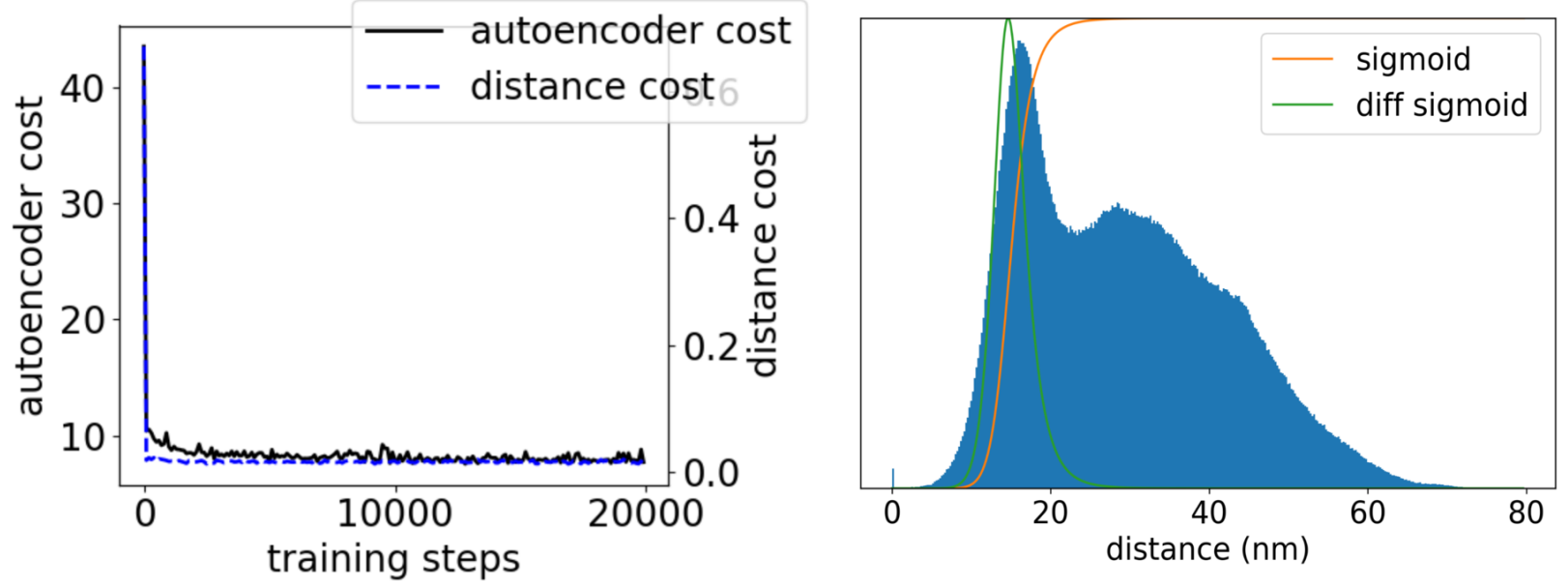

Figure S8.3: Illustration of parameters used to train an EncoderMap neural network on RMD data from 3xUb CG simulations. Distance distribution (blue) of high dimensional data (RMD, 288D) and resulting sigmoid function (orange) and its first derivative (green). Green graph indicates window where distances should be preserved during dimensionality reduction. 


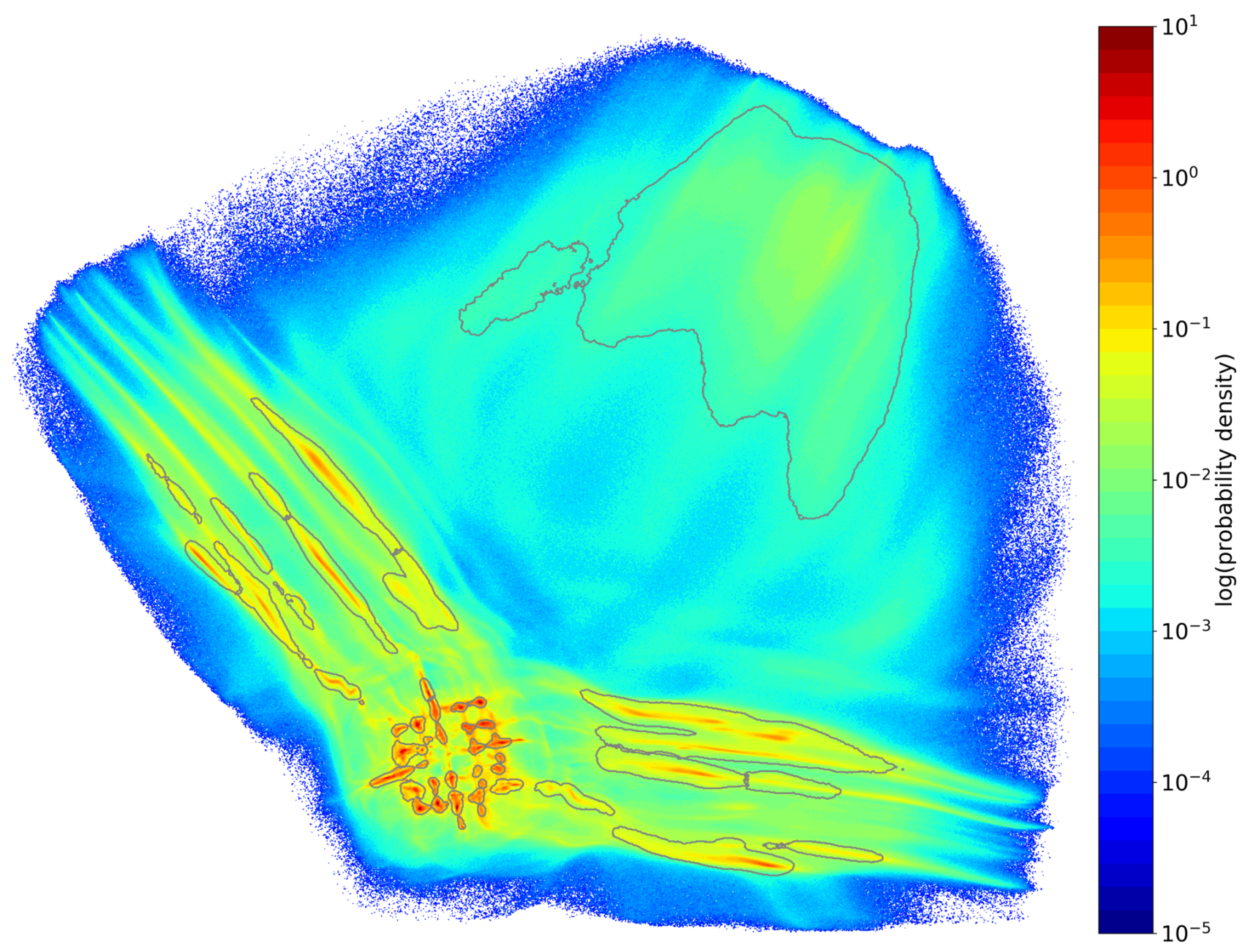

Figure S8.4: Larger version of Figure 8A. Configurational landscape of 3xUb (coloring according to the logarithm of the probability density). Histogram was obtained from projected (2D) RMD data of three unlinked Ub proteins. 

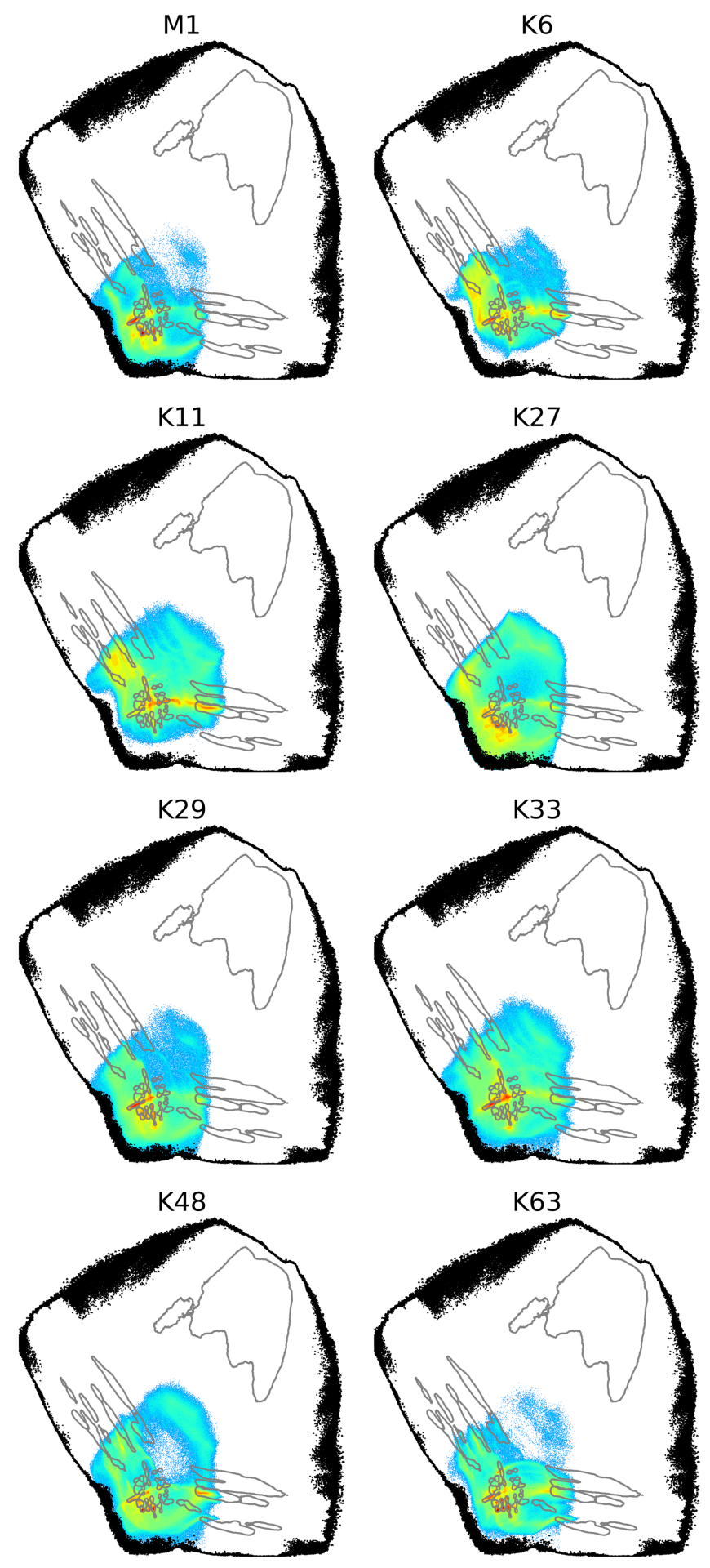

Figure S9.1: EncoderMap projections of triUb. Two-dimensional representations/landscapes separated by linkage type (colored histograms) with outer rim of 3xUb landscape (black) and outlines of clusters found for 3xUb (grey). Coloring according to the logarithm of the probability density. Color-bar according to Figure 9. 

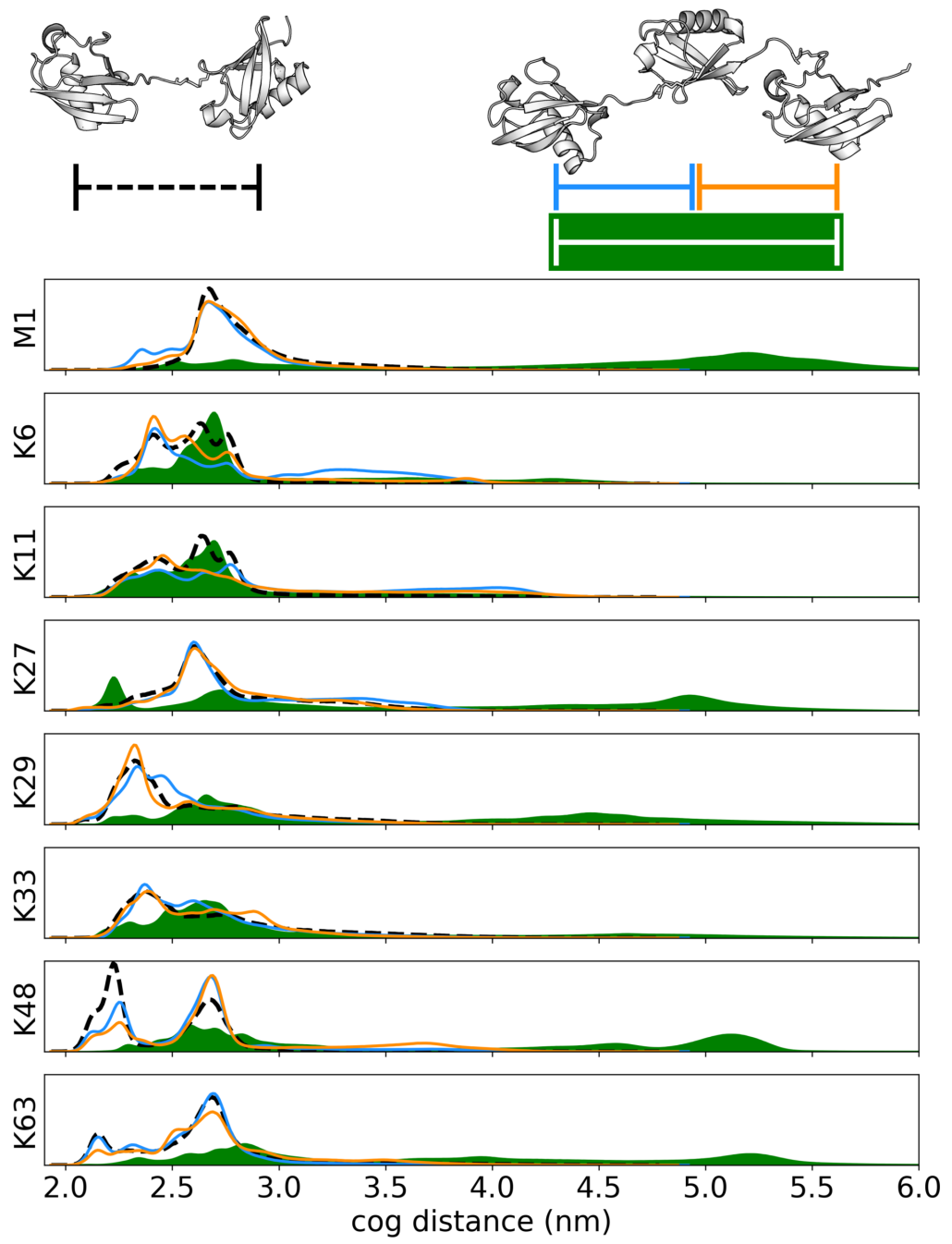

Figure S9.2: Distance distributions in diUb and triUb. Histogram of center of geometry distances calculated between Ub moieties in diUb (black) and adjacent moieties in triUb (blue, orange) and the distal and proximal moiety in triUb (end-to-end, green). 